\title{
Group Sparsity Based Multi-Target Tracking in Multi-Static Passive Radar Systems Using Doppler-Only Measurements
}

\author{
Saurav Subedi ${ }^{\dagger}$, Yimin D. Zhang ${ }^{\dagger}$, Moeness G. Amin ${ }^{\dagger}$, and Braham Himed ${ }^{\ddagger}$ \\ $\dagger$ Center for Advanced Communications, Villanova University, Villanova, PA 19085, USA \\ $\ddagger$ RF Technology Branch, Air Force Research Lab (AFRL/RYMD), WPAFB, OH 45433, USA
}

\begin{abstract}
In this paper, we consider the problem of multitarget tracking in a multi-static passive radar system using Doppler-only measurements. In a multi-static configuration, the observability and estimation accuracy of target states can be significantly improved by simultaneously exploiting all available measurements. Track-before-fuse and fuse-before-track are the two fusion paradigms proposed in the literature to utilize such multi-static measurements. The fuse-before-track approach involves a minimal information loss and thus achieves a better accuracy and robustness than the track-before-fuse counterpart. However, despite the obvious advantages in terms of estimation accuracy and robustness, the centralized measurement fusion approach is difficult due to the prohibitive computational cost. As such, the track-before-fuse approach has been commonly used in multi-static passive radar tracking systems using Doppleronly measurements. In this paper, we exploit a group-sparsity based algorithm to simultaneously utilize the Doppler shift measurements at all bistatic pairs to obtain the target state estimates directly in Cartesian coordinate system. The estimated target states at each sampling instant are then fed as the inputs to the linear Gaussian mixture probability hypothesis filter, which removes the false measurements and correctly associates the measurements to the respective targets. Simulation results are provided to validate the ability of the proposed method to successfully handle the multi-target tracking problem in a challenging environment characterized by missed detection and false measurements.
\end{abstract}

\section{INTRODUCTION}

Multi-target tracking (MTT) is a well investigated problem (e.g., [1-4]) relevant to diverse application areas including air traffic control, intelligence, surveillance, and reconnaissance (ISR), space applications, remote sensing, biomedicine, and robotics. MTT refers to a problem of jointly estimating the number of targets and their states, at successive time intervals, from a noisy and cluttered set of observations [5]. In recent years, the problem of MTT using Doppler-only measurements has emerged as an area of interest, specially in the context of multi-static passive radar (MPR) systems (e.g., [6-8]), as the Doppler sensors have become increasingly accurate and inexpensive. A Doppler-only tracking passive radar system being considered comprises an illuminator of opportunity (e.g., DAB/DVB broadcast station, FM radio transmitter, and

\footnotetext{
${ }^{\dagger}$ The work of S. Subedi, Y. D. Zhang, and M. G. Amin was supported in part by a subcontract with Defense Engineering Corporation for research sponsored by the Air Force Research Laboratory under Contract FA8650-12-D-1376.
}

cellular mobile transmitter), a distributed network of Doppler sensors that collect independent measurements in the form of Doppler shift of the target-reflected signals, and an information fusion center. Alternatively, a network comprising multiple spatially-separated transmitters and a single Doppler sensor can be deployed. Doppler-only tracking offers two key advantages in terms of ease-of-implementation and system expenses because (a) it does not require a strictly synchronous operation among the sensors, and (b) the required volume of information exchange, or the bandwidth, for communication among the sensors and/or between each sensor and the information fusion center is significantly reduced. On the other hand and from a signal processing perspective, there are several challenges associated with the Doppler-only MTT, such as measurement origin uncertainties, track initiation and management, data association, clutter, and detection loss [13].

There are several algorithms available in the literature that address the problem of multi-target measurement association and track management. Some of the common data association algorithms are multiple hypothesis tracking (MHT) [14], probabilistic data association (PDA) [15], and joint probabilistic data association (JPDA) [16]. These data association based algorithms suffer from exponentially increasing computational complexity as the number of targets increases. The probability hypothesis density (PHD) filter, based on a random finite set (RFS) framework and Bayesian analysis [2], avoids data association and, thus, has emerged as a computationally efficient alternative in MTT, particularly for problems involving a large number of targets. There are two algorithms available for the implementation of the PHD filter: the sequential Monte Carlo method (SMCPHD) and the Gaussian Mixture representation (GMPHD). The GMPHD algorithm provides a closed-form solution and is computationally efficient in linear dynamic systems, while the SMCPHD algorithm is more suited to the non-linear target state dynamics [7]. Several recent works (e.g., [6-8]) have investigated the application of the PHD filter for multi-target tracking using Doppler-only measurements.

In a multi-static configuration comprising multiple spatially distributed illuminators or receivers, the observability and accuracy of target state estimation can be significantly improved by simultaneously exploiting all available measurements. Also, when a target crosses the baseline between one of the transmitter-receiver pairs, the information available from 
other bistatic pairs can be exploited for reliable estimation, provided that there are a sufficient number of bistatic pairs in operation. In this paper, we consider the multiple receive sensor scenario, although the multiple illuminator case can be similarly formulated. There are two different fusion approaches available in the literature, namely, track-before-fuse and fuse-before-track. The track-before-fuse approach is a distributed algorithm where tracking is performed locally at each sensor, followed by a track-to-track fusion at a fusion center. On the other hand, the fuse-before-track approach is a centralized mechanism that involves a fusion of measurements from multiple sensors, followed by a tracking mechanism [9]. It is well established that the latter approach involves a minimal information loss and achieves better accuracy and robustness (e.g., [9-11]). However, due to a prohibitively high computational load associated with the existing centralized measurement fusion for MTT using Doppler-only measurements [8], most of the existing works related to Doppler-only target tracking in multi-static networks rely on the traditional track-before-fuse approach. For example, a random receiver selection algorithm is adopted in [6], whereas a sequential updating scheme is proposed in [12]. Nonetheless, there are four critical issues associated with these existing procedures: (a) The accuracy of the decentralized track-before-fuse method is generally lower than the centralized counterpart; (b) The target state remains unobservable until at least three Doppler measurements from spatially separated sensors are observed in a two-dimensional (2-D) tracking problem; (c) The entire tracking process is adversely affected due to error propagation, if the first few measurements provide poor bistatic Doppler information; and (d) More complex and computationally demanding tracking algorithms, such as extended Kalman filter (EKF)-based GMPHD or SMCPHD, are required to process highly non-linear Doppler-shift measurements.

In this paper, we present a novel measurement fusion scheme based on the group sparsity observed in an MPR system for MTT using Doppler-only measurements. We consider a situation where the sensors report a set of scalar and lowrate Doppler-shift measurements, and rather than the entire raw measurements, to the fusion center. We propose a groupsparsity based sparse signal reconstruction approach to fuse the information available in all bistatic links by exploiting the sparsity of the target position and motion parameters in a discretized position-velocity space. Using the groupsparsity based approach, multiple target states, in terms of the 2-D Cartesian coordinates of the position and velocity, are estimated at each sampling instant and are directly fed as instantaneous measurements to the GMPHD filter. This avoids the use of a non-linear measurement model, allowing a simple and computationally efficient Kalman filterbased implementation of the GMPHD filter. The GMPHD filter removes the false measurements and correctly associates the target-generated measurements to the respective targets. Simulation results are provided to validate the ability of the proposed method to successfully handle the multi-target tracking problem in a challenging environment characterized by missed detection and false measurements. The optimal subpattern assignment (OSPA) distance [17], which represents the sum of the localization error and the cardinality error, is used as an evaluation criterion to analyze the performance of the tracking filter.

Notations: A lower (upper) case bold letter denotes a vector (matrix). Specifically, $\mathbf{I}_{N}$ and $\mathbf{0}_{N}$ denote the $N \times N$ identity and zero matrices, respectively. $(.)^{*},(.)^{T}$, and $(.)^{H}$, respectively, denote complex conjugation, transpose, and hermitian operations. $\mathbb{R}^{n \times 1}$ and $\mathbb{C}^{n \times 1}$, respectively, represent the $n$ dimensional real and complex vectors. $\|\cdot\|$ denotes the $l_{2}$ norm of a vector, whereas $\Re($.$) and \Im($.$) , respectively, stand for the$ real and imaginary parts of a complex number, and $\mathcal{N}(x ; a, b)$ denotes variable $x$ to be a Gaussian distributed with mean $a$ and variance $b$. In addition, $\operatorname{diag}($.$) and \operatorname{tr}($.$) , respectively,$ denote the diagonal and trace operations.

\section{Signal Model}

We consider the problem of tracking multiple ground moving targets in an MPR system. The MPR network comprises a single broadcast station transmitting at a known carrier frequency $f_{c}$ and $N$ spatially distributed Doppler sensors. The transmitter is assumed to be located at $\mathbf{b}$, whereas the $n$th receiver is located at $\mathbf{r}^{(n)}, n=1, \ldots, N$. The transmitter and the receivers are assumed stationary and their locations are precisely known a priori at the fusion center.

We consider $T$ targets moving within the surveillance region. The state vector of the $i$ th target at the $k$ th observation, $\mathbf{x}_{k, i}$, represents a point in the state space $\mathcal{X} \in \mathbb{R}^{4 \times 1}$ and comprises its instantaneous position $\mathbf{p}_{k, i} \triangleq\left[p_{x, k, i}, p_{y, k, i}\right]^{T}$ and velocity $\mathbf{v}_{k, i} \triangleq\left[v_{x, k, i}, v_{y, k, i}\right]^{T}$ in the 2-D Cartesian coordinate system, i.e.,

$$
\mathbf{x}_{k, i}=\left[\mathbf{p}_{k, i}^{T}, \mathbf{v}_{k, i}^{T}\right]^{T} .
$$

The target dynamics is commonly modeled as a linear Gaussian constant velocity model [6], such that

$$
\mathbf{x}_{k, i}=\mathbf{F} \mathbf{x}_{k-1, i}+\mathbf{w}_{k, i}
$$

where $\mathbf{F}$ is the state transition matrix defined as

$$
\mathbf{F}=\left[\begin{array}{cc}
\mathbf{I}_{2} & \Delta \mathbf{I}_{2} \\
\mathbf{0}_{2} & \mathbf{I}_{2}
\end{array}\right]
$$

$\Delta$ is the sampling interval, $\mathbf{I}_{n}$ and $\mathbf{0}_{n}$, respectively, represent the $n \times n$ identity and zero matrices, and $\mathbf{w}_{k, i} \sim \mathcal{N}(\mathbf{0}, \mathbf{Q})$ is the process noise modeled as additive white Gaussian noise. The process noise covariance is defined as

$$
\mathbf{Q}=\sigma_{\mathbf{w}}^{2}\left[\begin{array}{cc}
\frac{\Delta^{4}}{4} \mathbf{I}_{2} & \frac{\Delta^{3}}{2} \mathbf{I}_{2} \\
\frac{\Delta^{3}}{2} \mathbf{I}_{2} & \Delta^{2} \mathbf{I}_{2}
\end{array}\right],
$$

where $\sigma_{\mathbf{w}}^{2}$ is the variance of the process noise.

The bistatic Doppler-shift corresponding to the target state $\mathbf{x}_{k, i}$ measured at the $n$th sensor is obtained as [18]

$$
f_{k, i}^{(n)}=-\frac{\mathbf{v}_{k, i}^{T}}{\lambda}\left[\frac{\mathbf{p}_{k, i}-\mathbf{r}^{(n)}}{\left\|\mathbf{p}_{k, i}-\mathbf{r}^{(n)}\right\|}+\frac{\mathbf{p}_{k, i}-\mathbf{b}}{\left\|\mathbf{p}_{k, i}-\mathbf{b}\right\|}\right]+e_{k, i}
$$

where $\lambda=c / f_{c}$ is the wavelength of the transmitted signal, $c$ is the velocity of propagation of a radio signal in free space and $e_{k, i}$ is the measurement error modeled as $\mathcal{N}\left(0, \sigma_{e}^{2}\right)$. The Doppler measurement space or the field-of-view is defined 
over an interval $\left[-f_{0},+f_{0}\right][8]$, where $f_{0}$ denotes the maximum possible Doppler shift.

In a multi-sensor network, each sensor measures a different Doppler-shift for the motion of the same target, depending on its bistatic configuration. Unlike [19-21], where entire raw measurement vectors corresponding to each bistatic link are assumed available at the fusion center, it is not possible to directly combine these scalar Doppler-shift measurements across different bistatic links deploying the fuse-before-track approach. Therefore, the existing tracking methods rely on the sub-optimal track-before-fuse schemes. However, it is noted that the Doppler-shift measurements share the same target state. As a result, it is possible to combine the information across all available bistatic links by exploiting the sparsity in a discretized position-velocity space. In a recent work [22], the $a$ priori information regarding sparsity is exploited in developing a hierarchical Bayesian Kalman filter, which basically combines the sparse Bayesian learning and Kalman filter, where the signal to be tracked is assumed to be sparse in its natural basis. In the following, we propose a method to simultaneously utilize the Doppler-shift measurements available at all sensors by exploiting the group sparsity of the measurements in a discretized position-velocity space.

\section{MEASUREMENT FUSION USING GROUP SPARSITY}

Let $\mathcal{F}_{k}^{(n)}=\left\{f_{k, 1}^{(n)}, \cdots, f_{k, T}^{(n)}\right\}$ be the set of Doppler-shift measurements at the $n$th sensor observed at the $k$ th observation interval corresponding to the $T$ targets. Since the Doppler measurements corresponding to multiple sensors share the same set of target states, i.e., the position and velocity of targets, as the common sparse support, we exploit the group sparsity based technique to effectively fuse the Doppler-shift measurements corresponding to all available bistatic links.

Each measurement can be represented as an impulse in a discrete Fourier space and, as such, the Doppler spectrum can be expressed as a linear sum of impulses in the frequency domain as

$$
Y_{k}^{(n)}(f)=\sum_{i=1}^{T} \delta\left(f-f_{k, i}^{(n)}\right),
$$

where $F_{s}$ is the sampling frequency and $-F_{s} / 2 \leq f \leq F_{s} / 2$. Taking the inverse fourier transform, we obtain the timedomain waveform as

$$
y_{k}^{(n)}(t)=\sum_{i=1}^{T(k)} \exp \left(-\jmath 2 \pi f_{k, i}^{(n)} t\right),
$$

where $0 \leq t \leq \Delta$ and $\jmath=\sqrt{-1}$. It is assumed that the target motion is rectilinear and uniform within an observation interval.

In order to apply a group sparsity based approach, we define a $\mathbb{C}^{N_{s} \times 1}$ observation vector for the $k$ th observation instant as

$$
\mathbf{y}_{k}^{(n)}=\left[y_{k}^{(n)}(1), \cdots, y_{k}^{(n)}\left(N_{s}\right)\right]^{T},
$$

where $N_{s}=\Delta \times F_{s}$ represents the number of discrete samples. The entire target state space is represented by a 4-D discrete space, where both position and velocity are measured in a 2D Cartesian coordinate system. Let an $N_{p x} N_{p y} N_{v x} N_{v y} \times 1$ vector $\mathbf{u}_{k}^{(n)}$ be the unknown sparse vector which vectorizes the discretized 4-D position-velocity space such that the $j$ th element of $\mathbf{u}_{k}^{(n)}$ is associated with the $j$ th hypothetical target state vector $\mathbf{x}_{k, j}=\left[\mathbf{p}_{k, j}^{T}, \mathbf{v}_{k, j}^{T}\right]^{T}$, where $\mathbf{p}_{k, j}=\left[p_{x, k, j}, p_{y, k, j}\right]^{T}$ and $\mathbf{v}_{k, j}=\left[v_{x, k, j}, v_{y, k, j}\right]^{T}$. The corresponding hypothetical bistatic Doppler-shift measurement at the $n$th sensor is given as

$$
f_{k, j}^{(n)}=-\frac{\mathbf{v}_{k, j}^{T}}{\lambda}\left[\frac{\mathbf{p}_{k, j}-\mathbf{r}^{(n)}}{\left\|\mathbf{p}_{k, j}-\mathbf{r}^{(n)}\right\|}+\frac{\mathbf{p}_{k, j}-\mathbf{b}}{\left\|\mathbf{p}_{k, j}-\mathbf{b}\right\|}\right],
$$

and following (8), we define an $N_{s} \times 1$ column vector

$$
\mathbf{y}_{k, j}^{(n)}=\left[y_{k, j}^{(n)}(1), \cdots, y_{k, j}^{(n)}\left(N_{s}\right)\right]^{T},
$$

where

$$
y_{k, j}^{(n)}(t)=\exp \left(-\jmath 2 \pi f_{k, j}^{(n)} t\right) .
$$

The column vector $\mathbf{y}_{k, j}^{(n)}$ comprises the $j$ th column of the $N_{s} \times N_{p x} N_{p y} N_{v x} N_{v y}$ dictionary matrix $\Psi_{k}^{(n)}$ defined for the $n$th bistatic link. Since we deal with complex data, we adopt a commonly used technique to decompose the complex observation into its real and imaginary parts, and rewrite the $n$th observation vector as

$$
\tilde{\mathbf{y}}_{k}^{(n)}=\left[\Re\left(\mathbf{y}_{k}^{(n)}\right)^{T}, \Im\left(\mathbf{y}_{k}^{(n)}\right)^{T}\right]^{T},
$$

and the corresponding dictionary matrix as

$$
\tilde{\mathbf{\Psi}}_{k}^{\prime(n)}=\left[\begin{array}{cc}
\Re\left(\boldsymbol{\Psi}_{k}^{(n)}\right) & -\Im\left(\boldsymbol{\Psi}_{k}^{(n)}\right) \\
\Im\left(\mathbf{\Psi}_{k}^{\prime(n)}\right) & \Re\left(\mathbf{\Psi}_{k}^{(n)}\right)
\end{array}\right],
$$

Thus, we can reformulate the underlying problem into a realvalued expression as

$$
\tilde{\mathbf{y}}^{(n)}=\tilde{\boldsymbol{\Psi}}^{(n)} \mathbf{u}^{(n)},
$$

where the sparse vectors $\mathbf{u}^{(n)}, n=1, \cdots, N$, share a common sparse support because the same set of target state is shared among all $N$ bistatic links. As such, the underlying problem can be formulated as a group sparse problem [23]. There are a number of algorithms available to solve the group sparse problems such as group basis pursuit [24], group LASSO [25], block orthogonal matching pursuit [26]. Multitask Bayesian compressive sensing algorithm $[27,28]$ provides an adaptive learning framework and generally outperforms conventional compressive sensing algorithms. In this paper, we use the complex multi-task Bayesian compressive sensing (CMT-BCS) algorithm [28], which is based on the Bayesian framework that exploits the statistical relationship between multiple measurements or sensing tasks and that between the real and imaginary parts of the sparse entries.

For every observation interval $k$, the solution of the CMTBCS algorithm converges to an $\hat{T}(k)$-sparse solution, whose indices correspond to estimates of $\hat{T}(k)$ state vectors, $\hat{\mathbf{x}}_{k, i}=$ $\left[\hat{p}_{x, k, i}, \hat{p}_{y, k, i}, \hat{v}_{x, k, i}, \hat{v}_{y, k, i}\right]^{T}$, where $i=1, \cdots, \hat{T}(k)$. A safe threshold is used in the CMT-BCS algorithm to relax the sparsity constraint, such that, the estimated number of targets may be larger than the actual number of targets, i.e., $\hat{T}(k) \geqslant T$. At every $k$, a set of these $\hat{T}(k)$ target state estimates is sent to the MTT filter, i.e.,

$$
Z_{k}=\left\{\mathbf{z}_{k, 1}, \cdots, \mathbf{z}_{k, \hat{T}(k)}\right\}
$$


where $\mathbf{z}_{k, i}=\hat{\mathbf{x}}_{k, i}, i=1, \cdots, \hat{T}(k)$. As such, the tracking filter is required to remove the false measurements and properly identify and associate the true target-generated measurements to the respective targets.

In the following, we use the RFS based filtering to tackle the problem of dynamically estimating multiple targets in the presence of false measurements and association uncertainty in the Bayesian filtering framework [5].

\section{RANDOM FINITE SET BASED FILTERING}

In the RFS framework, we define a set-valued state and set-valued measurement at the $n$th sensor, respectively, as

$$
X_{k}=\left\{\mathbf{x}_{k, 1}, \cdots, \mathbf{x}_{k, T}\right\},
$$

and

$$
Z_{k}=\left\{\mathbf{z}_{k, 1}, \cdots, \mathbf{z}_{k, \hat{T}(k)}\right\},
$$

where $T$ is the actual number of targets and the $\hat{T}(k)$ is the estimated number of targets at the $k$ th observation. An RFS model for the time evolution of a multi-target state $X_{k}$ at time $k$ from $X_{k-1}$ is defined as [5]

$$
X_{k}=\left[\bigcup_{\zeta \in X_{k}} S_{k \mid k-1}(\boldsymbol{\zeta})\right] \bigcup \Gamma_{k},
$$

where $S_{k \mid k-1}(\boldsymbol{\zeta})$ represents the RFS of the surviving targets and $\Gamma_{k}$ is the RFS of the spontaneous target births at $k$. The corresponding RFS measurement model observed at the $k$ th observation can be expressed as [5]

$$
Z_{k}=K_{k} \bigcup\left[\bigcup_{\mathbf{x} \in X_{k}} \Theta_{k}(\mathbf{x})\right],
$$

where $K_{k}$ is the RFS of the false measurements and $\Theta_{k}(\mathbf{x})$ is the RFS of the target-generated measurements.

The multi-target posterior density $p_{k}\left(. \mid Z_{1: k}\right)$, conditioned on the sets of measurements up to time $k$, can be determined using the Bayesian recursion [6]

$$
\begin{gathered}
p_{k \mid k-1}\left(X_{k} \mid Z_{1: k-1}\right)=\int f_{k \mid k-1}\left(X_{k} \mid X\right) p_{k-1}\left(X \mid Z_{1: k-1}\right) d X, \\
p_{k}\left(X_{k} \mid Z_{1: k}\right)=\frac{g_{k}\left(Z_{k} \mid X_{k}\right) p_{k \mid k-1}\left(X_{k} \mid Z_{1: k-1}\right)}{\int g_{k}\left(Z_{k} \mid X\right) p_{k \mid k-1}\left(X \mid Z_{1: k-1}\right) d X},
\end{gathered}
$$

where $f_{k \mid k-1}\left(X_{k} \mid X\right)$ is the multi-target transition density and $g_{k}\left(Z_{k} \mid X_{k}\right)$ is the multi-target likelihood. The optimal multitarget Bayesian recursion involves multi-set integrals which are computationally infeasible. The PHD filter provides a computationally efficient alternative by propagating the firstorder statistical moment of the multi-target state, rather than the multi-target posterior density. As such, the PHD recursion is defined as $[5,6]$

$$
\begin{aligned}
\nu_{k \mid k-1}(\mathbf{x})=\int p_{S} f_{k \mid k-1}(\mathbf{x} \mid \boldsymbol{\zeta}) \nu_{k-1}(\boldsymbol{\zeta}) d \boldsymbol{\zeta}+\gamma_{k}(\mathbf{x}), \\
\nu_{k}(\mathbf{x})=\left(1-p_{D}\right) \nu_{k \mid k-1}(\mathbf{x}) \\
+\sum_{\mathbf{z} \in Z_{k}} \frac{p_{D} g_{k}(\mathbf{z} \mid \mathbf{x}) \nu_{k \mid k-1}(\mathbf{x})}{\kappa_{k}(\mathbf{z})+\int p_{D} g_{k}(\mathbf{z} \mid \boldsymbol{\xi}) \nu_{k \mid k-1}(\boldsymbol{\xi}) d \boldsymbol{\xi}},
\end{aligned}
$$

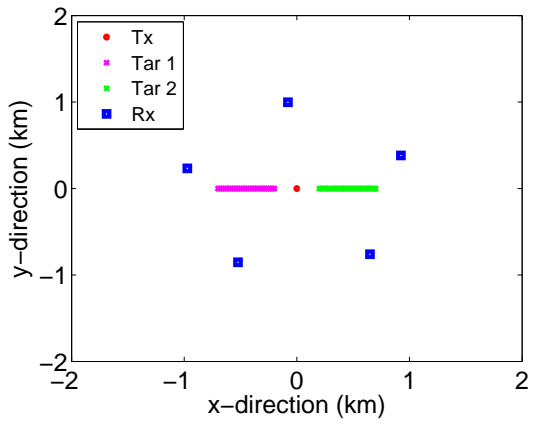

Fig. 1. Multi-static network configuration and true trajectories of the targets.

where $p_{S}$ is the probability of target survival, $\gamma_{k}(\mathbf{x})$ is the intensity of spontaneous birth RFS at time $k, \kappa_{k}(\mathbf{z})$ is the intensity of the clutter RFS at time $k$, and $p_{D}$ is the probability of target detection.

The GMPHD filter provides a closed-form solution to the PHD filter derived in [5]. It is noted that, unlike the existing works (e.g., $[6,8]$ ) that require an EKF based adaptation of the GMPHD filter in order to handle the Doppler-shift measurement based non-linear measurements, we can directly implement the linear Gaussian multi-target models discussed in [5].

\section{SIMULATION RESULTS}

In the simulations, we consider a geo-location scenario as illustrated in Fig. 1, where a broadcast station is located at the origin and transmitting at $950 \mathrm{MHz}$, and 5 Dopplershift measurement sensors are distributed along a circle of radius $1 \mathrm{~km}$ from the transmitter. We assume that the Doppler measurements are updated every $2 \mathrm{~s}$ at each sensor and the overall tracking duration is considered to be $50 \mathrm{~s}$. We consider two targets, initially located at $[-200,0]^{T} \mathrm{~m}$ and $[200,0]^{T} \mathrm{~m}$, moving away from each other in anti-parallel directions with a speed of $[-10,0]^{T} \mathrm{~m} / \mathrm{s}$ and $[10,0]^{T} \mathrm{~m} / \mathrm{s}$, respectively, as illustrated in Fig. 1.

The standard deviation of the additive white Gaussian noise associated with the Doppler shift measurements is assumed to be $\sigma_{e}=0.1 \mathrm{~Hz}$. For the implementation of the GMPHD filter, we consider the probability of target survival to be $p_{S}=0.99$ for both targets. Each target follows a linear Gaussian dynamics as defined in (2). The sampling period $\Delta$ is considered to be $2 \mathrm{~s}$, and the initial value of the standard deviation of the process noise, $\sigma_{\mathbf{w}}$, is assumed to be $0.2 \mathrm{~m} / \mathrm{s}^{2}$. A Poisson RFS $\Gamma_{k}$ with intensity

$$
\gamma_{k}(X)=0.1 \mathcal{N}\left(m_{\gamma}^{(1)}, P_{\gamma}\right)+0.1 \mathcal{N}\left(m_{\gamma}^{(2)}, P_{\gamma}\right),
$$

is used to model spontaneous births in the vicinity of $m_{\gamma}^{(1)}$ and $m_{\gamma}^{(2)}$, where $m_{\gamma}^{(1)}=[-250,0,-15,0]^{T}, m_{\gamma}^{(2)}=$ $[250,0,15,0]^{T}$ and $P_{\gamma}=\operatorname{diag}\left([100,100,100,100]^{T}\right)$. Each target is assumed to be detected with a probability of $p_{D}=$ 0.98 .

The output of the group-sparsity based target state estimation, described in section III, is shown in Fig. 2 for each sampling instant. It can be observed that, by exploiting the 


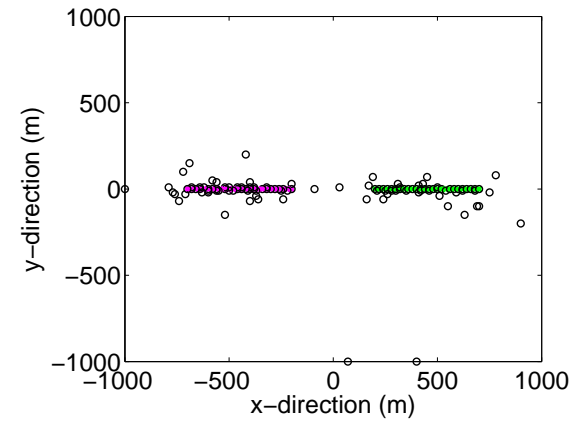

Fig. 2. Instantaneous target positions estimated from group sparse reconstruction.

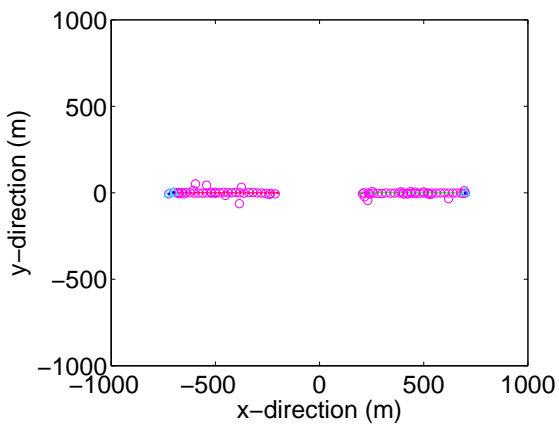

Fig. 3. Results of the GMPHD filter.

group sparsity of the multi-static Doppler shift measurements in the discretized position-velocity space, the proposed method for target state estimation obtains estimations closely grouped around the actual target trajectory throughout the observation period. However, since the number of targets in the observation scene is unknown, a safe threshold is chosen to relax the sparsity constraint in the CMT-BCS algorithm. As a result, we observe some false measurements distributed over the observation scene.

The estimated target states obtained at each sampling instant are fed into the GMPHD filter, which has the following tasks: (a) discerning false measurements from target-generated measurements and removing them; (b) performing multi-target measurement association; and (c) reducing the position error for each track or smoothing the estimated track. The estimated trajectories of the targets are shown in Fig. 3, which verify that the GMPHD filter successfully discards the false estimates that appear as outliers from the actual target trajectory.

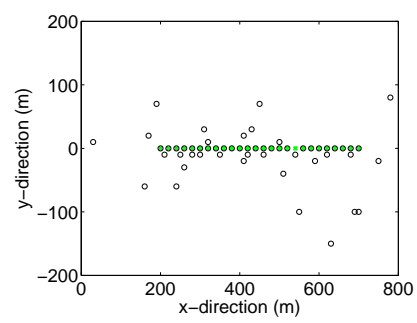

(a) Group sparsity based estimation

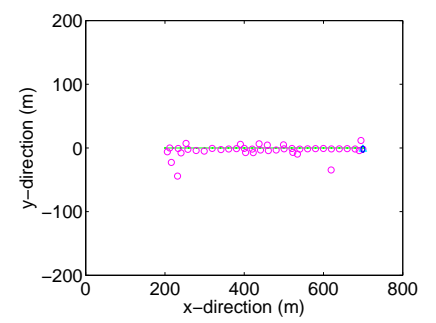

(b) GMPHD tracking result
Fig. 4. Illustration of performance of GMPHD filter.

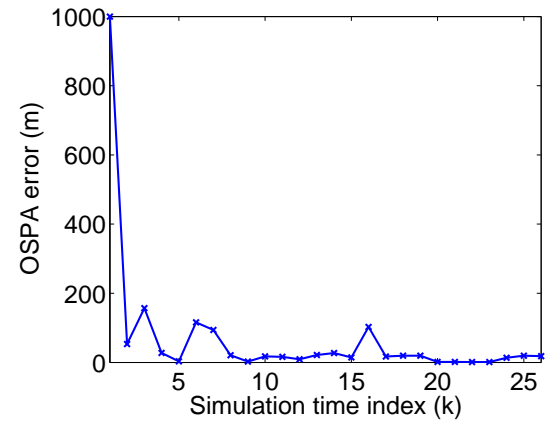

Fig. 5. OSPA results.

In order to demonstrate that the tracking filter successfully handles the remaining two tasks of multi-target measurement association and reducing the position error, we enlarge the trajectory of one of the targets in Fig. 4. It is evident that, at the steady state, the tracking filter reduces the overall cardinality and position errors by exploiting the estimations obtained from the group sparsity based signal reconstruction. The performance of the filter, in terms of the OSPA error, is shown in Fig. 5. The OSPA error between the ground truth state set $X_{k}$ and the estimated state set $\hat{X}_{k}$ with cardinalities $m$ and $n$, respectively, is defined as $[6,17]$

$d_{p, \beta}\left(X_{k}, \hat{X}_{k}\right)=\left(\min _{\pi \in \Pi_{m}} \frac{1}{m} \sum_{i=1}^{n} d_{\beta}\left(\mathbf{x}_{i}, \hat{\mathbf{x}}_{\pi i}\right)^{p}+\frac{\beta^{p}}{m}(m-n)\right)^{\frac{1}{p}}$

if $m \leq n$, and $d_{p, \beta}\left(X_{k}, \hat{X}_{k}\right)=d_{p, \beta}\left(\hat{X}_{k}, X_{k}\right)$ if $n>m$, where $p<\infty$ is the OSPA metric order parameter, and $d_{\beta}\left(\mathbf{x}_{i}, \hat{\mathbf{x}}_{\pi i}\right)=\min \left(\beta, d\left(\mathbf{x}_{i}, \hat{\mathbf{x}}_{\pi i}\right)\right)$ is the cut-off distance between two tracks, with $d\left(\mathbf{x}_{i}, \hat{\mathbf{x}}_{\pi i}\right)$ being the base distance error between the two tracks and $\beta>0$ being the cut-off parameter which determines the relative weight given to the cardinality error against the base distance error. The variable $\Pi_{m}$ represents the set of all permutations of length $m$ with elements taken from $1, \cdots, n$. In this simulation, we use $p=1$ and $\beta=1000$, respectively. It is observed that initially it takes sometime for the GMPHD filter to detect the presence of the targets and associate the measurements with the respective targets. After some observations, the filter correctly detects and closely tracks the targets, providing an accurate tracking performance.

\section{CONCLUSions}

In this paper, we have developed a fusion scheme based on the signal group sparsity available in all bistatic links in an MPR system for MTT using Doppler-only measurements. The estimates of the multiple target states, i.e., 2-D Cartesian coordinates of position and velocity, are directly fed as instantaneous measurements to a Kalman filter-based implementation of the GMPHD filter. Simulation results verified a successful implementation of a multi-target tracking problem in a challenging environment and the performance of the GMPHD filter was evaluated using the OSPA error metric. 


\section{REFERENCES}

[1] Y. Bar-Shalom, "Tracking methods in a multitarget environment," IEEE Transactions on Automatic Control, vol. 23, no. 4, pp. 618-626, Aug. 1978.

[2] R. P. S. Mahler, "Multitarget Bayes filtering via first-order multitarget moments," IEEE Transactions on Aerospace and Electronic Systems, vol. 39, no. 4, pp. 1152-1178, Oct. 2003.

[3] B. Vo, S. Singh, and A. Doucet, "Sequential Monte Carlo methods for multitarget filtering with random finite sets," IEEE Transactions on Aerospace and Electronic Systems, vol. 41, no. 4, pp. 1224-1245, Oct. 2005.

[4] C. Hue, J. P. Le Cadre, and P. Perez, "Tracking multiple objects with particle filtering," IEEE Transactions on Aerospace and Electronic Systems, vol. 38, no. 3, pp. 791812, Jul. 2002.

[5] B. Vo and W. K. Ma, "The Gaussian mixture probability hypothesis density filter," IEEE Transactions on Signal Processing, vol. 54, no. 11, pp. 4091-4104, Nov. 2006.

[6] M. B. Guldogan, D. Lindgren, F. Gustafsson, H. Habberstad, and U. Orguner, "Multi-target tracking with PHD filter using Doppler-only measurements," Digital Signal Processing, vol. 27, pp. 1-11, Apr. 2014.

[7] J. H. Yoon, D. Y. Kim, S. Bae, and V. Shin, "Joint initialization and tracking of multiple moving objects using Doppler information," IEEE Transactions on Signal Processing, vol. 59, no. 7, pp. 3447-3452, Jul. 2011.

[8] B. Ristic and A. Farina, "Target tracking via multi-static Doppler shifts," IET Radar, Sonar and Navigation, vol. 7, no. 5, pp. 508-516, Jun. 2013.

[9] B. Shapo and C. Kreucher, "Track-before-fuse error bounds for tracking passive targets," in Proceedings of International Conference on Information Fusion (FUSION), Chicago, IL, Jul. 2011.

[10] S. Sun and Z. Deng, "Multi-sensor optimal information fusion Kalman filter," Automatica, vol. 40, no. 6, pp. 1017-1023, Jun. 2004.

[11] C. Kreucher and B. Shapo, "Multitarget detection and tracking using multisensor passive acoustic data," IEEE Journal of Oceanic Engineering, vol. 36, no. 2, pp. 205218, Apr. 2011.

[12] N. T. Pham, W. Huang, and S. H. Ong, "Multiple sensor multiple object tracking with GMPHD filter," in Proceedings of International Conference on Information Fusion (FUSION), Quebec City, Canada, Jul. 2007.

[13] M. Mallick, B. N. Vo, T. Kirubarajan, and S. Arulampalam, "Introduction to the issue on multitarget tracking," IEEE Journal of Selected Topics in Signal Processing, vol. 7, no. 3, pp. 373-375, Jun. 2013.

[14] S. S. Blackman, "Multiple hypothesis tracking for multiple target tracking," IEEE Aerospace and Electronic Systems Magazine, vol. 19, no. 1, pp. 5-18, Jan. 2004.

[15] D. Musicki, R. Evans, and S. Stankovic, "Integrated probabilistic data association," IEEE Transactions on Au- tomatic Control, vol. 39, no. 6, pp. 1237-1241, Jun. 1994.

[16] T. E. Fortmann, Y. Bar-Shalom, and M. Scheffe, "Sonar tracking of multiple targets using joint probabilistic data association," IEEE Journal of Oceanic Engineering, vol. 8, no. 3, pp. 173-184, Jul. 1983.

[17] D. Schuhmacher, B. T. Ho, and B. N. Ho, "A consistent metric for performance evaluation of multi-object filters," IEEE Transactions on Signal Processing, vol. 56, no. 8, pp. 3447-3457, Aug. 2008.

[18] Y. D. Zhang and B. Himed, "Moving target parameter estimation and SFN ghost rejection in multistatic passive radar," in Proceedings of IEEE Radar Conference, Ottawa, Canada, April 2013.

[19] S. Subedi, Y. D. Zhang, M. G. Amin, and B. Himed, "Robust motion parameter estimation in multistatic passive radar," in Proceedings of European Signal Processing Conference, Marrakech, Morocco, Sept. 2013.

[20] S. Subedi, Y. D. Zhang, M. G. Amin, and B. Himed, "Motion parameter estimation of multiple targets in multistatic radar through sparse signal recovery," in Proceedings of IEEE International Conference on Acoustics, Speech, and Signal Processing (ICASSP), Florence, Italy, May 2014.

[21] S. Subedi, Y. D. Zhang, M. G. Amin, and B. Himed, "Motion parameter estimation of multiple ground moving targets in multi-static passive radar systems," EURASIP Journal on Advances in Signal Processing, vol. 2014, no. 157, pp. 1-14, Oct. 2014.

[22] E. Karseras, K. Leung, and W. Dai, "Tracking dynamic sparse signals using Hierarchical Bayesian Kalman filters," Proceedings of IEEE International Conference on Acoustics, Speech, and Signal Processing (ICASSP), Vancouver, Canada, May 2013.

[23] Y. C. Eldar, P. Kuppinger, and H. Bolcskei, "Block-sparse signals: uncertainty relations and efficient recovery," IEEE Transactions on Signal Processing, vol. 58, no. 6, pp. 3042-3054, Jun. 2010.

[24] E. V. D. Berg and M. Friedlander, "Probing the pareto frontier for basis pursuit solutions," SIAM Journal on Scientific Computing, vol. 31, no. 2, pp. 890-912, 2008.

[25] M. Yuan and Y. Lin, "Model selection and estimation in regression with grouped variables," Journal of the Royal Statistical Society Series B, vol. 68, no. 1, pp. 49-67, 2006.

[26] L. Zelnik-Manor, K. Rosenblum, and Y. C. Eldar, "Sensing matrix optimization for block-sparse decoding," IEEE Transactions on Signal Processing, vol. 59, no. 9, pp. 4300-4312, Sept. 2011.

[27] S. Ji, D. Dunson, and L. Carin, "Multitask compressive sensing," IEEE Transactions on Signal Processing, vol. 57, no. 1, pp. 92-106, Jan. 2009.

[28] Q. Wu, Y. D. Zhang, M. G. Amin, and B. Himed, "Complex multi-task bayesian compressive sensing", in Proceedings of IEEE International Conference on Acoustics, Speech, and Signal Processing (ICASSP), Florence, Italy, May 2014. 